\title{
A Joint Resource Allocation Algorithm for D2D Communication
}

\author{
Abdul Kadir Hamid ${ }^{1}$, Lamia Osman Widaa ${ }^{2}$, Fahd N. Al-Wesabi ${ }^{3, *}$, Imran Khan ${ }^{4}$, Anwer Mustafa \\ Hilal $^{5}$, Manar Ahmed Hamza ${ }^{5}$, Abu Sarwar Zaman ${ }^{5}$ and Mohammed Rizwanullah
}

\author{
${ }^{1}$ Department of Electrical \& Computer Engineering, University of Sharjah, Sharjah, United Arab Emirates \\ ${ }^{2}$ Department of Electrical Engineering, College of Engineering, Princess Nourah bint Abdulrahman University, \\ Saudi Arabia \\ ${ }^{3}$ Department of Computer Science, King Khalid University, Muhayel Aseer, KSA \& Faculty of Computer and IT, Sana'a \\ University, Yemen \\ ${ }^{4}$ Department of Electrical Engineering, University of Engineering and Technology Peshawar, Pakistan \\ ${ }^{5}$ Department of Computer and Self Development, Preparatory Year Deanship, Prince Sattam bin Abdulaziz University, \\ AlKharj, Saudi Arabia \\ *Corresponding Author: Fahd N. Al-Wesabi. Email: falwesabi@kku.edu.sa \\ Received: 11 May 2021; Accepted: 25 June 2021
}

\begin{abstract}
The emergence of multimedia services has meant a substantial increase in the number of devices in mobile networks and driving the demand for higher data transmission rates. The result is that, cellular networks must technically evolve to support such higher rates, to be equipped with greater capacity, and to increase the spectral and energy efficiency. Compared with $4 \mathrm{G}$ technology, the $5 \mathrm{G}$ networks are being designed to transmit up to 100 times more data volume with devices whose battery life is 10 times longer. Therefore, this new generation of networks has adopted a heterogeneous and ultra-dense architecture, where different technological advances are combined such as device-to-device (D2D) communication, which is one of the key elements of $5 \mathrm{G}$ networks. It has immediate applications such as the distribution of traffic load (data offloading), communications for emergency services, and the extension of cellular coverage, etc. In this communication model, two devices can communicate directly if they are close to each other without using a base station or a remote access point. Thus, eliminating the interference between the $\mathrm{D} 2 \mathrm{D}$ and cellular communication in the network. The interference management has become a hot issue in current research. In order to address this problem, this paper proposes a joint resource allocation algorithm based on the idea of mode selection and resource assignment. Simulation results show that the proposed algorithm effectively improves the system performance and reduces the interference as compared with existing algorithms.
\end{abstract}

Keywords: D2D communication; resource allocation; wireless networks; mobile communication 


\section{Introduction}

In recent years, wireless local area networks (WLANs) have become quite popular due to their low cost of deployment and easy implementation, which enables the rapid proliferation of personal networks. But some unfavorable points should not be overlooked such as the operation in frequency bands where there is no interference control or guaranteed safety control [1-3]. These aspects motivate the inclusion of a new communication channel in mobile networks: Device-toDevice communication (D2D), which is currently under investigation to be included in the LTEAdvanced standard. The creation of IMT-Advanced promotes the application of new technologies that satisfy their requirements, from which the LTE-Advanced arises and begins to be standardized at the end of 2009 [4-9]. The LTE corresponds to the name of the commercial standard developed by the 3rd Generation Partnership Project (3GPP) for the evolution of UMTS mobile terrestrial radio access networks (e-UTRAN) standardized as of Release 8. D2D communication enables the establishment of a peer-to-peer communication mode between two LTE-A terminals, in such a way that there would be a better use of the LTE network resources and a higher throughput would be provided to the terminals in D2D communication [10].

In order to improve the performance of cellular networks and meet the user's demand for speed, related technologies to improve the utilization of spectrum resources have become the research hotspots, such as cognitive radio and D2D communication technologies [11-14]. In traditional cellular networks, data is transmitted through base stations or other network elements. By using the D2D communication technology, when user terminals are relatively close, data can be directly transmitted. It cannot only increase the system throughput but also reduces the equipment power consumption and improves the system's spectrum utilization [15,16]. It also brings new challenges to wireless resource management, especially when D2D users reuses the cellular user resources, there is relatively serious co-channel interference. In order to reduce the co-channel interference, some interference management schemes have been proposed one after another [1720]. Reference [21] proposes a power control scheme to ensure the SINR of the cellular link. Reference [22] proposes an optimal power allocation scheme in single-cell user and single D2D pair scenarios. Reference [23] proposes a game-based joint scheduling and resource allocation algorithm. Reference [24] proposes a resource allocation scheme based on interference perception. However, the above studies assumed that, the D2D pair reuses one cellular user resource. In fact, in downlink transmission, when the D2D pair is far from the eNB, the D2D pair can reuse one or more cellular user resources, thereby further improving the system throughput. When the D2D pair is close to the eNB, it suffers from greater interference and can use dedicated resources for communication.

This paper proposes a joint mode selection and resources allocation scheme based on the location of D2D users in the cell. In this method, the eNB first classifies the D2D pair according to the distance from the eNB. When the D2D pair is closer to the eNB, it is considered as a nearend D2D pair, and the eNB preferentially allocates the dedicated resources. When the D2D pair is far from the eNB, it is a remote D2D pair, and the eNB preferentially allocates the multiplexing resources. As the cycle progresses, the eNB will dynamically adjust the resource allocation for the D2D pair according to the resources and the terminal link rate, so that the D2D pair can be allocated appropriate resources. The simulation results show that the proposed scheme effectively reduces the interference between the users and improves the system throughput. 


\section{System Model}

Assuming that the eNB in the macro cell is in the center of the cell, the cell contains two types of users, namely D2D and cellular users. D2D users exist in pairs, where each pair includes a transmitting and a receiving terminal. The D2D and cellular users are randomly distributed in the cell. The distance between the D2D transmitting and receiving terminals in the same D2D pair meets the maximum distance constraint, thereby ensuring the quality-of-service (QoS). Fig. 1 shows the scenario of downlink transmission. Assuming that, there is $C$ number of cellular user equipment (CUE), $K$ number of D2D pairs in the macro cell, and the position of the D2D pairs in the cell does not change.

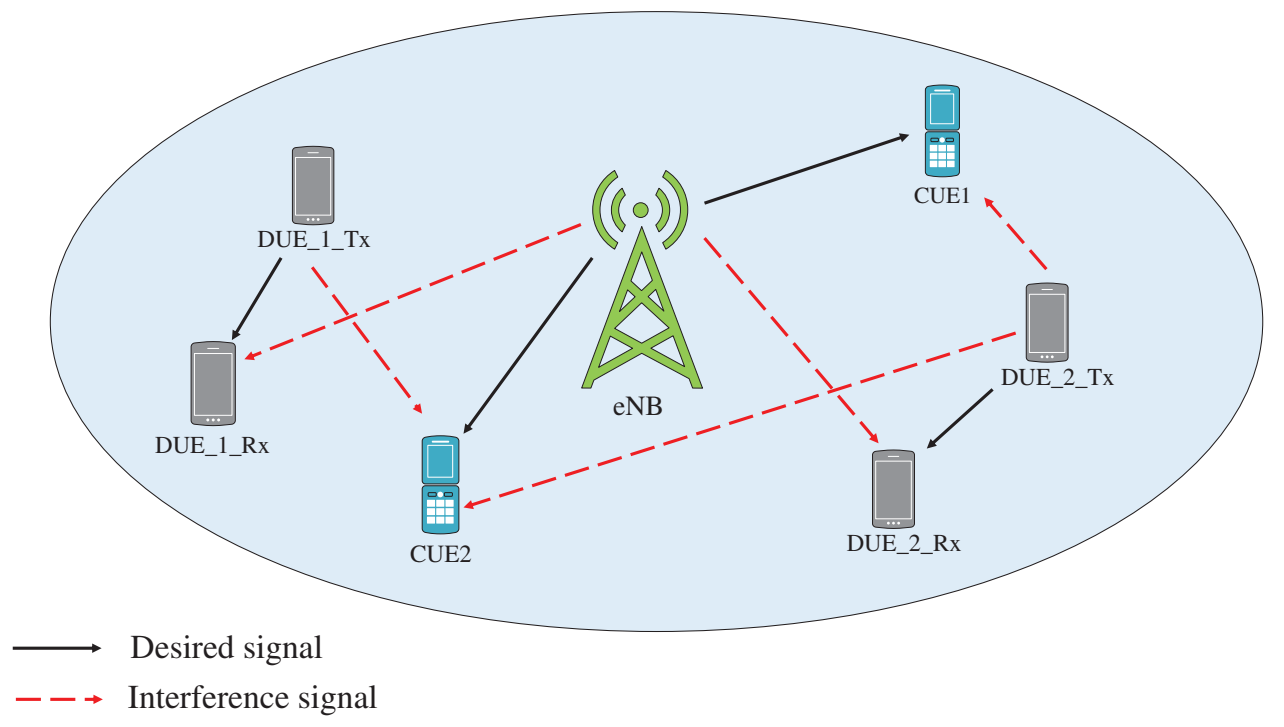

Figure 1: Proposed system model

According to the distance between the eNB and receiving terminal of the D2D pair, the eNB divides the user pair into two types, namely the near-end D2D pair close to the eNB and the far-end D2D pair far away from the eNB. Assuming that, there are $N$ orthogonal sub-channels $(N>C)$ in the cell with bandwidth $B$, and $n$ fixed sub-channels are allocated to the cellular users. Define a set of binary variables $\left\{x_{k, n}\right\}$ to represent the use of sub-channels for D2D users. When $x_{k, n}=1$, the D2D user uses the sub-channel $n$ for $k$. When $x_{k, n}=0$, the D2D user does not uses the sub-channel $n$ for $k$. The D2D pair in the scheme has two communication modes, namely multiplexing mode and dedicated mode. In the multiplexing mode, the D2D user communicates on multiplexing cellular sub-channels. In dedicated mode, the D2D pair uses a dedicated sub-channel for communication $\left(\sum_{n=C+1}^{N} \leq 1, \forall k \in\{1,2, \ldots, K\}\right)$, at the same time, each dedicated sub-channel can only be allocated to one D2D pair $\left(\sum_{k=1}^{K} \leq 1, \forall n \in\{C+1, C+2, \ldots, N\}\right)$. Assume that, the eNB and D2D working in multiplexing mode have fixed power for each cellular sub-channel transmission, which is $P_{\mathrm{B}}$ and $P_{\mathrm{D}}$ respectively. Since the D2D pair working in the dedicated mode will not cause interference to other users, therefore, it uses the maximum power $P_{\mathrm{D}}^{\max }$ for transmission. 
The SINR of a D2D pair working in the dedicated mode is expressed as:

$\gamma_{k, n}^{\mathrm{D}}=\frac{P_{\mathrm{D}}^{\max } G_{\mathrm{k}}^{\mathrm{D}}}{N_{0}}, \quad C+1 \leq n \leq N, 1 \leq k \leq K$

Among them, $G_{\mathrm{k}}^{\mathrm{D}}$ represents the channel gain between the $\mathrm{D} 2 \mathrm{D}$ pair $k, N_{0}$ represents the thermal noise power of the receiving terminal.

The SINR of a D2D pair working in the multiplexing mode is expressed as

$\gamma_{k, n}^{\mathrm{D}}=\frac{P_{\mathrm{D}} G_{\mathrm{k}}^{\mathrm{D}}}{I_{k, n}^{\mathrm{D}}+N_{0}}=\frac{P_{\mathrm{D}} G_{\mathrm{k}}^{\mathrm{D}}}{x_{k, n} P_{\mathrm{B}} G_{\mathrm{k}}^{\mathrm{B}}+\sum_{j \in\left\{j \mid x_{j, n}=1 \cap j \neq k\right\}} P_{\mathrm{D}} G_{j, k}^{\mathrm{D}}+N_{0}}, \quad 1 \leq n \leq C, \quad 1 \leq k, \quad j \leq K$

where, $G_{\mathrm{k}}^{\mathrm{B}}$ represents the channel gain between the receiving terminal of D2D pair $k$ and the eNB; $G_{j, k}^{\mathrm{D}}$ represents the channel gain between the transmitting D2D pair $j$ and the receiving D2D pair $k ; I_{k, n}^{\mathrm{D}}=x_{k, n} P_{\mathrm{B}} G_{\mathrm{k}}^{\mathrm{B}}+\sum_{j \in\left\{j \mid x_{j, n}=1 \cap j \neq k\right\}} P_{\mathrm{D}} G_{j, k}^{\mathrm{D}}$ is the interference when using sub-channel $n$ for $k$. The interference is divided into two parts: one part is generated by eNB, and the other part is generated by D2D pair using the same sub-channel $n$.

The rate when the $\mathrm{D} 2 \mathrm{D}$ user uses the sub-channel $n$ for $k$ is

$\eta_{k, n}^{\mathrm{D}}=B \log \left(1+\gamma_{k, n}^{\mathrm{D}}\right), \quad 1 \leq n \leq N, \quad 1 \leq k \leq K$

The link transmission rate of $\mathrm{D} 2 \mathrm{D}$ users to $k$ is expressed as

$\eta_{k}^{\mathrm{D}}=\sum_{n \in\left\{n \mid x_{k, n}=1\right\}} B \log \left(1+\gamma_{k, n}^{\mathrm{D}}\right)$

The SINR of CUE can be expressed as

$\gamma_{k, n}^{\mathrm{D}}=\frac{P_{\mathrm{B}} G_{n}^{C}}{I_{n}^{C}+N_{0}}=\frac{P_{\mathrm{B}} G_{n}^{C}}{\sum_{k \in\left\{k \mid x_{k, n}=1\right\}} P_{\mathrm{D}} G_{k, n}^{C}+N_{0}}, \quad 1 \leq n \leq C, \quad 1 \leq k \leq K$

Among them, $G_{n}^{C}$ represents the channel gain between the CUE $n$ and eNB, $G_{k, n}^{C}$ represents the channel gain between the transmitting terminal of D2D pair $k$ and CUE $n$, and $I_{n}^{C}$ represents the interference received by CUE $n$.

The link rate of CUE $n$ is expressed as:

$\eta_{n}^{C}=B \log \left(1+\gamma_{n}^{C}\right)$

The total throughput of the system is expressed as:

$\eta_{\mathrm{all}}=\sum_{n=1}^{C} \eta_{n}^{C}+\sum_{k=1}^{K} \eta_{k}^{C}$

In the downlink, the interference in the multiplexing mode mainly comes from the eNB and the D2D pair using the same sub-channel. Since the transmit power of the eNB is much greater 
than the transmit power of the D2D terminal, when the D2D pair and eNB are closer, then the interference is greater. At the same time, when the D2D pairs using the same sub-channel are closer, the interference between them is also greater. Therefore, the user pairs are classified according to the distance between the D2D pair and the eNB. When $d_{k} \leq d_{\text {th }}$ (where $d_{\text {th }}$ is the judgment threshold, $d_{k}$ is the distance from the D2D pair $k$ to the eNB), the D2D pair $k$ is the near-end D2D and the eNB preferentially allocates the dedicated resources. When $d_{k}>d_{\mathrm{th}}$, the D2D pair $k$ is the remote D2D pair, and the eNB preferentially allocates the cellular user resources to it.

In practice, the value of $d_{k}$ can be obtained from the pilot signal receiving power sent by the eNB, such as the reference signal receiving power (RSRP). Therefore, $d_{\text {th }}$ can correspond to a power threshold $P_{\text {th }}$. Suppose that, the D2D pair $k$ receives the reference signal received power from the eNB as $P_{k}$. When $P_{k} \geq P_{\text {th }}$, the D2D pair $k$ is the near-end D2D pair. Otherwise, it is considered as the far-end D2D pair.

\section{Proposed Algorithm}

This paper proposes a joint mode selection and resources allocation scheme based on the position of the D2D pair in the cell. The algorithm is divided into 3 stages, namely, the initial stage, dedicated resource allocation stage, and the multiplexed resource allocation stage. After the initial phase is completed, the dedicated resource allocation phase and the multiplexed resource allocation phase will be cycled. Through continuous cycles, the eNB will allocate appropriate resources to the D2D pair.

\subsection{Initialization}

When the communication link of the D2D pair is established, the reference signal from the eNB is first received and compared with the threshold $P_{\text {th }}$, and the result is fed back to the eNB. The eNB classifies the D2D pair according to the feedback information.

If the D2D pair $k$ reference signal received power $P_{k}$ satisfies $P_{k} \geq P_{\text {th }}$, then the D2D pair $k$ belongs to the near-end D2D pair set $S_{1}$, otherwise, it belongs to the far-end D2D pair set $S_{0}$, namely

$D U E_{k} \in \begin{cases}S_{1}, & P_{k} \geq P_{\text {th }} \\ S_{0}, & P_{k}<P_{\text {th }}\end{cases}$

During initialization, the near-end D2D pair works in a dedicated mode and uses the dedicated resources. The far-end D2D pair works in a multiplexing mode and reuses the cellular user resources. As the cycle progresses, the eNB dynamically allocate the resources to the D2D pair according to the resources and link rate.

\subsection{Allocation of Dedicated Resources}

In the dedicated resource allocation phase, the scheduler allocates the dedicated sub-channels in proper order. In the first dedicated resource allocation stage, the number of cycles $t=1$. At the beginning of scheduling, the first confirm dedicated D2D pair set $\mathrm{S}_{1}$ participates in the scheduling, that is, the D2D pair scheduled in this stage is composed of the near-end D2D pair $S_{1}$ and the $\mathrm{D} 2 \mathrm{D}$ pair whose terminal rate is less than the threshold in the multiplexing resource allocation stage. In each scheduling cycle, the scheduler will dedicate a sub-channel to the D2D pair with the highest priority (the priority of the D2D pair is calculated according to Eq. (9)). In this way, the 
dedicated sub-channels in the cell will be allocated to the D2D pair based on the priority. When the D2D pair $k$ is allocated to the dedicated sub-channel $n$, the scheduler will delete such pair and dedicate the sub-channel $n$ from set $S_{1}$ and dedicate the sub-channel set $U_{\text {sub }}^{D}$, respectively. When all dedicated sub-channels in the cell are allocated or all D2D pairs are scheduled, the dedicated resource allocation will be terminated. If the dedicated resource allocation is terminated, the set $S_{1}=\varnothing$ and the scheduler will add the D2D pair to the multiplexed set $S_{2}$, so that it can communicate through multiplexing cellular user resources. In this way, the system efficiency has been improved.

The priority function $Q_{k}(t)$ of the D2D pair is mainly composed of the estimated link rate $\eta_{k}^{D}(t)$ and the additional cost $C_{k}(t)$ of the D2D pair in the $t$-th cycle, which is expressed as follows:

$Q_{k}(t)=\eta_{k}^{D}(t)-C_{k}(t)=\eta_{k}^{D}(t)-\delta \sum_{t=t-1-m}^{t-1} \eta_{k}^{D}(t)$

Among them, the additional cost is expressed as $C_{k}(t)=\delta \sum_{t=t-1-m}^{t-1} \eta_{k}^{D}(t)$, which consists of the sum of the fairness coefficient $\delta(\delta \geq 0)$ and the link rate obtained by D2D pair $k$ in the first $m$ cycles, where $\eta_{k}^{D}(t)=0, t \in\{-m, \ldots, 0\}$. When $\delta$ is large, the sum of link rates obtained by D2D in the previous $m$ cycles of $\mathrm{D} 2 \mathrm{D}$ has a greater impact on the current priority calculation. Since there is no historical rate record of the D2D pair in the scheduler at the beginning of the cycle, the priority of the D2D pair is judged according to the estimated transmission rate in the first cycle, that is, $C_{k}(1)=0$.

\subsection{Allocation of Reused Resources}

Reusing the D2D pairs in the set $S_{2}$ will communicate through multiplexing the cellular subchannels, but it does not guarantee that the throughput of the entire system will increase. It is because, when the D2D pairs multiplexing the cellular sub-channels, it gets a certain rate gain, but suffers from certain interference, which affects the link rate, and vice versa. While considering the cellular rate and D2D's constraints on the maximum transmit power, better system throughput can be achieved through a reasonable allocation of resources. In the multiplexing resource allocation stage, the scheduling is performed in the form of tokens. In the first multiplexing resource allocation, the D2D pairs in the set $S_{2}$ do not reuse any cellular sub-channels. The resource allocation is summarized in the following steps.

Step 1: Confirm the multiplexed D2D pair set $S_{2}$ participating in the scheduling, that is, the pair scheduled in this stage is composed of the remote D2D pair and the pair that is not allocated to the dedicated sub-channel in the dedicated resource allocation stage.

Step 2: When the token is passed to the D2D pair $k$, it will check whether the cellular sub-channels are multiplexed by themselves one by one. If it is multiplexed, go to step 3; otherwise, go to step 4. If the D2D pair $k$ has detected all the cellular sub-channels $U_{\text {sub }}^{C}=$ $\left\{U_{\text {sub } 1}, U_{\text {sub2 }}, \ldots, U_{\text {sub } C}\right\}$, then the scheduler will delete the D2D pair $k$ from set $S_{2}$. If $S_{2}$ is not an empty set after deletion, the token will be passed to the next D2D pair. If $S_{2}$ is deleted, the loop will end, and the D2D pair whose link rate is lower than the threshold $R_{\text {th }}^{D}$ will be added to the set $S_{1}$.

Step 3: Suppose that, when the D2D pair $k$ multiplexes the cellular sub-channel $U_{\text {subn }}$, the rates of D2D pair $k$ and D2D pair $j\left(j \in\left\{j \mid x_{j, n}=1 \cap j \neq k\right\}\right)$ using the same sub-channel are $\eta_{k, n}^{D}$ 
and $\eta_{j, n}^{D}$ respectively. The link rate of CUE $n$ is $\eta_{n}^{C}$. If the D2D pair $k$ does not use the subchannel $n$, the rate of $\mathrm{D} 2 \mathrm{D}$ pair $j$ will become $\tilde{\eta}_{j, n}^{D}$ which is expressed in Eq. (10) as follows $\tilde{\eta}_{j, n}^{D}=B \log \left(1+\tilde{\gamma}_{j, n}^{D}\right)=B \log \left(1+\frac{P_{\mathrm{D}} G_{j}^{D}}{I_{j, n}^{D}-P_{\mathrm{D}} G_{k, j}^{D}+N_{0}}\right)$

Wherein, $\tilde{\gamma}_{j, n}^{D}$ represents the SINR of $j$ th user when $k$ th user do not uses the sub-channel $n ; I_{j, n}^{D}$ (see Eq. (2)) represents the interference of the D2D user to $j$ when its user uses the sub-channel $n$ for $k$.

When the D2D users multiplexes the cellular sub-channel $n$ to $j$, the rate change value is

$\Delta \tilde{\eta}_{j, n}^{D}=\tilde{\eta}_{j, n}^{D}-\eta_{j, n}^{D}, \quad j \in\left\{j \mid x_{j, n}=1 \cap j \neq k\right\}$

In the same way, the link rate of the CUE $n$ will become

$\tilde{\eta}_{n}^{C}=B \log \left(1+\tilde{\gamma}_{n}^{C}\right)=B \log \left(1+\frac{P_{\mathrm{B}} G_{n}^{C}}{I_{n}^{C}-P_{\mathrm{D}} G_{k, n}^{C}+N_{0}}\right)$

where $\tilde{\gamma}_{n}^{C}$ represents the SINR of CUE $n$ after D2D users releases the sub-channel $n$ to $k$; $I_{n}^{C}$ (see Eq. (5)) represents the interference to CUE $n$ when the D2D user uses the sub-channel $n$ for $k$. The link rate change value of CUE $n$ is expressed as

$\Delta \eta_{n}^{C}=\tilde{\eta}_{n}^{C}-\eta_{n}^{C}$

If $\eta_{k, n}^{D}, \Delta \eta_{j, n}^{D}, \Delta \eta_{n}^{C}, R_{\mathrm{th}}^{C}$ (cellular link rate threshold) meets the following

$\eta_{k, n}^{D}<\sum_{j \in\left\{j \mid x_{j, n}=1 \cap j \neq k\right\}} \Delta \eta_{j, n}^{D}+\Delta \eta_{n}^{C} \| \eta_{n}^{C}<R_{\mathrm{th}}^{C}$,

then the D2D pair $k$ will stop using the sub-channel $n$, otherwise, it will continue to use the sub-channel $n$. Go back to step 2 .

Step 4: Assume that, the D2D user do not reuse the sub-channels of CUE $n$ for $k$. First, determine whether the transmit power of $\mathrm{D} 2 \mathrm{D}$ to $k$ is less than the maximum transmit power $P_{D}^{\max }$. If the total transmit power of $\mathrm{D} 2 \mathrm{D}$ to $k$ is equal to the maximum transmit power $P_{D}^{\max }$, return to step 2, otherwise perform the following steps.

Assume that, the rate of the D2D pair $j \in\left\{j \mid x_{j, n}=1\right\}$ of multiple CUE $n$ sub-channels is $\eta_{j, n}^{D}$, the link rate of CUE $n$ is $\eta_{n}^{C}$. If the D2D users uses the sub-channel $n$ for $k$, the rate of D2D for $j$ will be $\tilde{\eta}_{j, n}^{D}$ which is as follows

$\tilde{\eta}_{n}^{D}=B \log \left(1+\tilde{\gamma}_{j, n}^{D}\right)=B \log \left(1+\frac{P_{\mathrm{D}} G_{j}^{D}}{I_{j, n}^{D}+P_{\mathrm{D}} G_{k, j}^{D}+N_{0}}\right)$ 
where, $\tilde{\gamma}_{j, n}^{D}$ represents the SINR of the D2D user to $j$ after using the sub-channel $n$ for $k ; I_{j, n}^{D}$ represents the interference of the D2D users to $j$ when $k$ th user do not use the sub-channel $n$. When the D2D pair multiplexes the cellular sub-channel $n$ to $j$, the rate change value is

$\Delta \eta_{j, n}^{D}=\eta_{n}^{D}-\tilde{\eta}_{n}^{D}, \quad j \in\left\{j \mid x_{j, n}=1\right\}$

In the same way, the link rate of the CUE $n$ will become

$\tilde{\eta}_{n}^{D}=B \log \left(1+\tilde{\gamma}_{n}^{C}\right)=B \log \left(1+\frac{P_{\mathrm{B}} G_{n}^{C}}{I_{n}^{C}+P_{\mathrm{D}} G_{k, n}^{C}+N_{0}}\right)$

Among them, $\tilde{\gamma}_{n}^{C}$ represents the SINR of CUE $n$ after D2D multiplexes $k$ to CUE $n ; I_{n}^{C}$ represents the interference to the CUE $n$ when the D2D uses the subchannel $n$ for $k$. The rate change value of the CUE $n$ is

$\Delta \eta_{n}^{C}=\eta_{n}^{C}-\tilde{\eta}_{n}^{C}$

If $\eta_{k, n}^{D}, \Delta \eta_{j, n}^{D}, \Delta \eta_{n}^{C}, R_{\mathrm{th}}^{C}$ (cellular link rate threshold) meets the following

$\eta_{k, n}^{D}>\sum_{j \in\left\{j \mid x_{j, n}=1\right\}} \Delta \eta_{j, n}^{D}+\Delta \eta_{n}^{C} \& \& \tilde{\eta}_{n}^{C}>R_{\mathrm{th}}^{C}$

then the D2D pair will uses the sub-channel $n$ for $k$, otherwise it will not be allocated the subchannel. Go back to step 2.

\section{Simulation Results}

A link-level simulation program is established to test the scheme, and the simulation results are compared with the D2D scheme of multiplexing a cellular sub-channel and the pure cellular scheme.

\subsection{Simulation Configuration and Parameters}

Consider a single-cell scenario, where the cellular users and D2D pairs are randomly distributed in the cell. The distance between the terminals in a D2D pair meets the maximum distance constraint for communication. The received power $P_{i}=P_{j} L_{i j}\left|h_{i j}\right|^{2}$, where $P_{i}$ and $P_{j}$ represents the received and transmit power, respectively. $L_{i j}$ represents the path loss between the transmitting and the receiving terminal. $h_{i j}$ represents the complex Gaussian channel coefficient, and $h_{i j} \sim \mathrm{CN}(0,1)$. For the cellular link, the path loss is calculated according to the COST-231 Hata model, and for the D2D link, the path loss is calculated according to the modified model of short-distance communication [25]. The specific parameters are shown in Tab. 1.

\subsection{Simulation Results}

The system throughput simulation results of different schemes are shown in Fig. 2. When the channel quality of the direct link is good, the system throughput of the proposed algorithm and D2D pair multiplexing a cellular sub-channel scheme is greater than that of the pure cellular scheme. Although the D2D communication brings an increase in the total system throughput by reusing the cellular resources, the gain in the total system throughput decreases as the distance between the D2D pair and the terminal increases. When the maximum distance between the D2D 
pair is $50 \mathrm{~m}$, the proposed scheme increases the performance by $41 \%$ as compared to the D2D pair multiplexing one cellular sub-channel scheme.

Table 1: Simulation parameters

\begin{tabular}{ll}
\hline Parameter & Value \\
\hline Number of near-end D2D pairs & $2 \sim 7$ \\
Number of remote D2D pairs & $2 \sim 7$ \\
Cell radius & $500 \mathrm{~m}$ \\
Number of cellular users in the cell & $6 \sim 21$ \\
eNB maximum transmit power & $46 \mathrm{dBm}$ \\
Maximum transmit power of the terminal & $23 \mathrm{dBm}$ \\
Noise spectral density & $-174 \mathrm{dBm} / \mathrm{Hz}$ \\
D2D link path loss & $31.54+40 \log _{10}(d)$ \\
Cellular link path loss & $36.7+35 \log _{10}(d)$ \\
D2D link maximum distance & $30 \sim 50 \mathrm{~m}$ \\
\hline
\end{tabular}

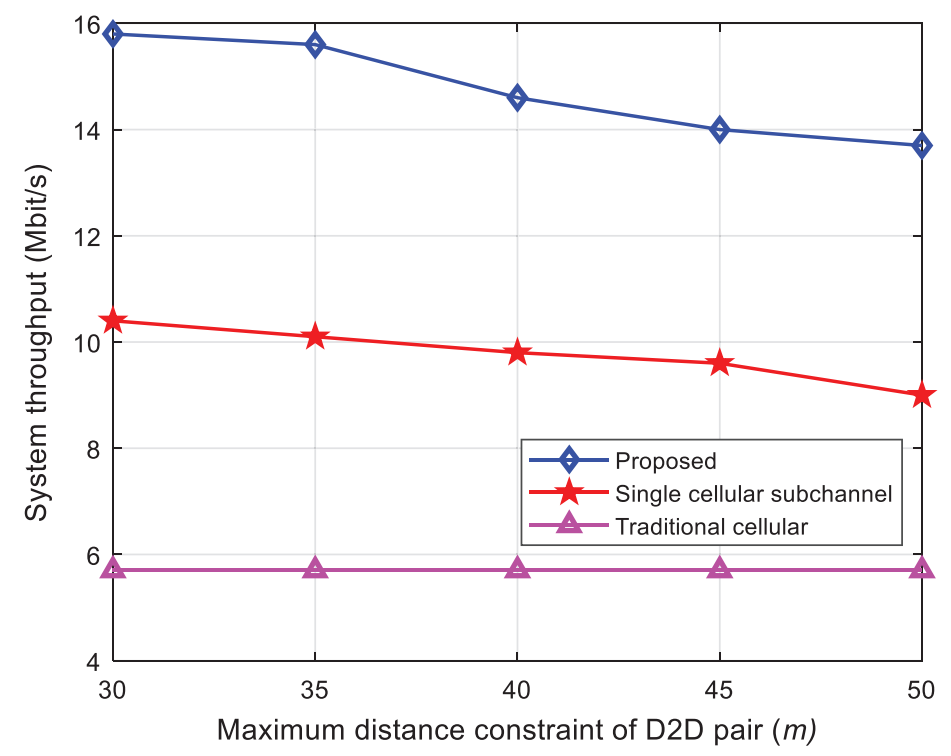

Figure 2: System throughput of different scenarios

Fig. 3 shows the simulation results of the average user rate of different scenarios. It can be seen from Fig. 3 that, the average user rate proposed scheme is higher than the single-cellular subchannel and the traditional cellular schemes. As the number of D2D pairs increases, the average user rate has also been improved in the proposed scheme whereas the single-cellular sub-channel and traditional schemes shows fixed average user rate.

The simulation results prove that the proposed scheme effectively reduces the interference between users and improves the system throughput. 


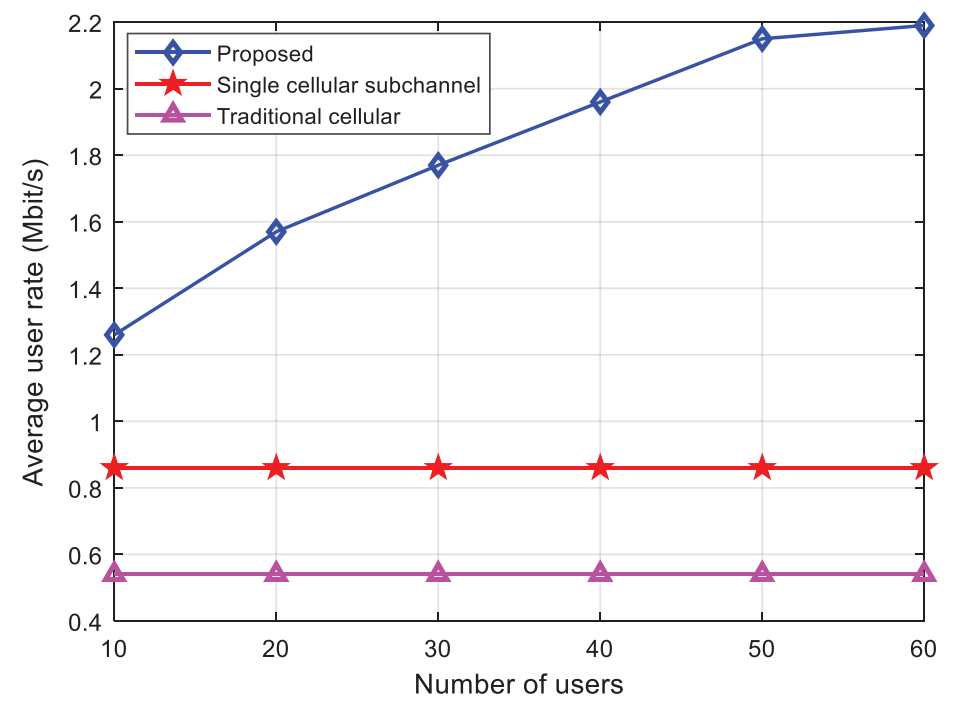

Figure 3: Average user rate of different schemes

Fig. 4 compared the average user rate of the algorithms vs. maximum transmit power. As can be seen from Fig. 4, the average user rate of all algorithms increases with increasing the transmit power. Moreover, the proposed algorithm gives a higher average user rate as compared with the existing algorithms. This shows that for all values of maximum transmit power, the proposed algorithm provides a better user rate and improves QoS.

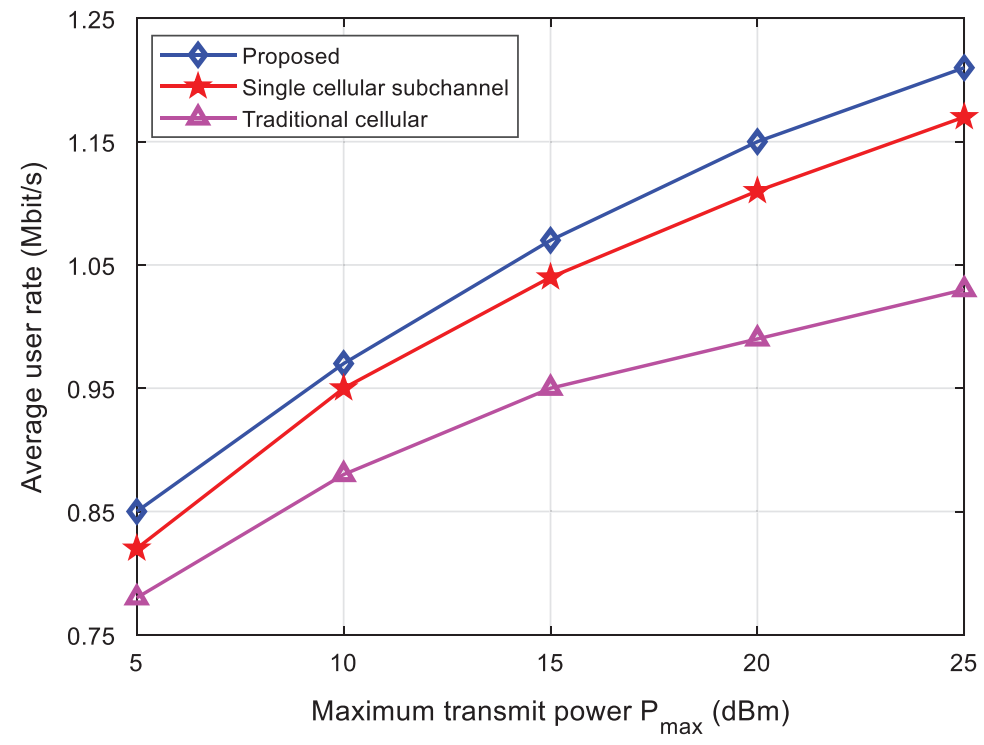

Figure 4: Comparison of average user rate vs. transmit power

\section{Conclusions}

This paper analyzes the interference between the cellular users and D2D users and proposes a joint resource allocation scheme. In the solution, the eNB optimizes the communication mode 
and resource allocation of the D2D users according to their location in the cell and the terminal interference. The simulation results show that, compared with the traditional cellular scheme and the D2D-pair multiplexing single-cellular sub-channel scheme, the proposed scheme can better improve the total system throughput and terminal link rate, and reduces the interference between the users. Further research as an extension to this work is to consider the QoS and energy efficiency aspects and evaluate the performance.

Availability of Data and Material: The data used for the findings of this study is available upon request.

Funding Statement: The authors extend their appreciation to the Deanship of Scientific Research at King Khalid University for funding this work under Grant Number (RGP.2/23/42), www.kku.edu.sa. This research was funded by the Deanship of Scientific Research at Princess Nourah bint Abdulrahman University through the Fast-track Research Funding Program.

Conflicts of Interest: The authors declare that they have no conflicts of interest to report regarding the present study.

\section{References}

[1] M. Waqas, Y. Niu, Y. Li, M. Ahmed, D. Jin et al., "A comprehensive survey on mobility-aware D2D communications: Principles, practice and challenges," IEEE Communications Surveys \& Tutorials, vol. 22, no. 3, pp. 1863-1886, 2020.

[2] M. Ahmed, Y. Li, M. Waqas, M. Sheraz, D. Jin et al., "A survey on socially aware device-to-device communications," IEEE Communications Surveys \& Tutorials, vol. 20, no. 3, pp. 2169-2197, 2018.

[3] F. S. Shaikh and R. Wismuller, "Routing in multi-hop cellular device-to-device (D2D) networks: A survey," IEEE Communications Surveys \& Tutorials, vol. 22, no. 3, pp. 2622-2657, 2018.

[4] M. Haus, M. Waqas, A. Y. Ding, Y. Li, S. Tarkoma et al., "Security and privacy in device-to-device (D2D) communication: A review," IEEE Communications Surveys \& Tutorials, vol. 19, no. 2, pp. 1054 1079, 2017.

[5] O. Hayat, R. Ngah, S. Z. M. Hashim, M. H. Dahri, R. F. Malik et al., "Device discovery in D2D communication: A survey," IEEE Access, vol. 7, pp. 131114-131134, 2019.

[6] A. Asadi, Q. Wang and V. Mancuso, "A survey on device-to-device communication in cellular networks," IEEE Communications Surveys \& Tutorials, vol. 16, no. 4, pp. 1801-1819, 2014.

[7] B. M. Elhalawany, R. Ruby and K. Wu, "D2D communication for enabling internet-of-things: Outage probability," IEEE Transactions on Vehicular Technology, vol. 68, no. 3, pp. 2332-2345, 2019.

[8] I. Loannou, V. Vassiliou, C. Christophorou and A. Pitsillides, "Distributed artificial intelligence solution for D2D communication in 5G networks," IEEE Systems Journal, vol. 14, no. 3, pp. 4232-4241, 2020.

[9] P. Pawar, A. Trivedi and M. K. Mishra, "Outage and ASE analysis for power controlled D2D communication,” IEEE Systems Journal, vol. 14, no. 2, pp. 2269-2280, 2020.

[10] S. Bashir, M. H. Alsharif, I. Khan, M. A. Albreem, A. Sali et al., "MIMO-Terahertz in 6G nanocommunications: Channel modeling and analysis," Computers, Materials \& Continua, vol. 66, no. 1, pp. 263-274, 2020.

[11] A. Amin, X. Liu, I. Khan, P. Uthansakul, M. Forsat et al., "A robust resource allocation scheme for device-to-device communications based on Q-learning," Computers, Materials \& Continua, vol. 65, no. 2, pp. 1487-1505, 2020.

[12] S. Alemaishat, O. A. Saraereh, I. Khan, S. H. Affes, X. Li et al., "An efficient precoding scheme for millimeter-wave massive MIMO systems," Electronics, vol. 8, no. 9, pp. 1-15, 2019. 
[13] A. Al-Nimrat, M. Smadi, O. A. Saraereh and I. Khan, "An efficient channel estimation scheme for mmwave massive MIMO systems," in Proc. IEEE Int. Conf. on Communication, Networks and Satellite, Makassar, Indonesia, pp. 1-8, 2019.

[14] I. Khan and D. Singh, "Efficient compressive sensing based sparse channel estimation for 5G massive MIMO systems," AEU-International Journal of Electronics and Communications, vol. 89, no. 2, pp. 181190, 2018.

[15] A. Amin, X. H. Liu, M. A. Saleem, S. Henna, T. Islam et al., "Collaborative wireless power transfer in wireless rechargeable sensor networks," Wireless Communications and Mobile Computing, vol. 15, pp. $1-13,2020$.

[16] F. Jameel, T. Ristaniemi, I. Khan and B. M. Lee, "Simultaneous harvest-and-transmit ambient backscatter communications under Rayleigh fading," EURASIP Journal on Wireless Communications and Networking, vol. 19, no. 1, pp. 1-9, 2019.

[17] W. Shahjehan, S. Bashir, S. L. Mohammed, A. B. Fakhri, A. A. Isaiah et al., "Efficient modulation scheme for intermediate relay-aided IoT networks," Applied Sciences, vol. 10, no. 6, pp. 1-12, 2020.

[18] O. A. Saraereh, A. Alsaraira, I. Khan and B. J. Choi, "A hybrid energy harvesting design for on-body internet-of-things (IoT) networks," Sensors, vol. 20, no. 2, pp. 1-14, 2020.

[19] T. Jabeen, Z. Ali, W. U. Khan, F. Jameel, I. Khan et al., "Joint power allocation and link selection for multi-carrier buffer aided relay network," Electronics, vol. 8, no. 6, pp. 1-15, 2019.

[20] S. Alemaishat, O. A. Saraereh, I. Khan and B. J. Choi, "An efficient resource allocation algorithm for D2D communications based on noma," IEEE Access, vol. 7, pp. 120238-120247, 2019.

[21] C. H. Yu, O. Tikkonen, K. Doppler and C. Ribeiro, "On the performance of device-to-device underlay communication with simple power control," in IEEE Vehicular Technology Conf., Barcelona, Spain, pp. $1-5,2009$.

[22] C. H. Yu, O. Tirkkonen, K. Doppler and C. Rebeiro, "Power optimization of device-to-device communication underlaying cellular communication," in IEEE Int. Conf. on Communications, Dresden, Germany, pp. 1-5, 2009.

[23] F. R. Wang, L. Y. Song, Z. Han, Q. Zhao and X. Wang, "Joint scheduling and resource allocation for device-to-device underlay communication," in IEEE Wireless Communications and Networking Conf., Shanghai, China, pp. 134-139, 2013.

[24] P. Janis, V. Koivunen, C. Ribeiro, J. Korhonen, K. Doppler et al., "Interference-aware resource allocation for device-to-device radio underlaying cellular networks," in IEEE Vehicular Technology Conf., Barcelona, Spain, pp. 1-5, 2009.

[25] S. Wen, X. Y. Zhu, X. Zhang and D. Yang, "QoS-aware mode selection and resource allocation scheme for device-to-device (D2D) communication in cellular networks," in IEEE Int. Conf. on Communications, Budapest, pp. 101-105, 2013. 